\title{
Anatomical and clinical justification of a minimally invasive technique for implantation an additional medial plate for bone osteosynthesis in patients with fractures of the distal femur
}

\author{
A.Iu. Kochish ${ }^{1,2}$, I.G. Belen'kii ${ }^{3}$, G.D. Sergeev ${ }^{1}$, B.A. Maiorov ${ }^{3,4}$ \\ ${ }^{1}$ Vreden National Medical Research Center of Traumatology and Orthopedics, Saint Petersburg, Russian Federation \\ ${ }^{2}$ Kirov Military Medical Academy, Saint Petersburg, Russian Federation \\ ${ }^{3}$ Pavlov First St. Petersburg State Medical University, Saint Petersburg, Russian Federation \\ ${ }^{4}$ Vsevolozhsk clinical inter-district hospital, Vsevolozhsk, Leningrad region, Russian Federation
}

\begin{abstract}
Introduction Traditional lateral plate osteosynthesis for distal femoral fractures does not provide sufficient stabilization in comminuted fractures due to a lack of medial support. Materials and methods Study on 16 lower limbs of 9 cadavers was conducted. We performed osteosynthesis of distal femur with precontoured medial plate implantation using a minimally invasive surgical technique. Then, the soft tissues were dissected to explore an exact location of the medial plate and the distances between plate and crucial anatomic structures of the distal femur. Fifteen patients underwent surgical treatment of distal femoral fractures with a loss of medial stability (AO/OTA classification 33-A3, 33-C2, 33-C3 fracture types) using the method of low invasive double-plating. Follow-up examinations were performed after 6 to 12 months. Results were evaluated using KSS and Lysholm scales. Results Cadaver study showed safety and feasibility of described minimally invasive osteosynthesis of distal femoral fractures method. We did not encounter specific complications associated with medial plate implantation during surgery. All fractures healed 12 months after surgery. The functional outcomes were good and excellent in more than $70 \%$ of cases according to KSS and Lysholm scales. Discussion Fracture management in the segments with eccentric axial load is still problematic. The double-plating technique is a promising method which can be used by trauma surgeons in distal femoral fractures without medial stability. However, further clinical research to prove safety and feasibility of this method of distal femoral fracture fixation in comparison with alternate ostheosynthesis techniques is needed to determine more specific indications for its application.

Keywords: distal femoral fracture, plate, osteosynthesis, minimally invasive plating
\end{abstract}

\section{INTRODUCTION}

Fractures of the distal femur (FDF) are rare but are always severe trauma. Their frequency is about $0.4 \%$ of all limb fractures. The affected are mosly young men around the age of 30 and older women over the age of 65 . The most common types of fractures under discussion are high-energy injuries due to road traffic accidents or sport activities at a young age and low-energy injuries from falls of one's own height in elderly patients with osteoporosis $[1,2]$.

Among all fractures of the femur, the incidence of FDFs ranges from 4 to $6 \%$ [3]. However, due to the peculiarities of the anatomy of the femur and knee joint, treatment of such an injury is a difficult task, and the rate of unsatisfactory treatment outcomes may reach $44 \%$ [4]. Among the complications of treatment, $6 \%$ are non-unions, $3.3 \%$ of cases are failure of fixation of bone fragments, and the rate of infectious complications reaches $2.7 \%$. Moreover, $16.8 \%$ of cases may need repeated surgical interventions [5].

In the current practice of managing such injuries, anatomically pre-curved plates with angular stability of screws are most often used for osteosynthesis in patients with FDF. The generally accepted technique for their implantation is a minimally invasive surgical technique, according to which the implant is introduced epiperiosteally from two small surgical approaches along the lateral surface of the thigh and fixed with screws to the femur [6, 7]. However, in complex multifragmented FDFs, there is a loss of medial stability in the area of damage to the femur as one laterally located plate does not provide sufficient stability for fixing bone fragments. Therefore, various options for additional medial fixation have been proposed $[7,8,9]$.

In particular, our earlier biomechanical experiments on plastic models of the femur showed a relatively high resistance to cyclic loads of the boneimplant system, which included two plates located on the lateral and medial sides of the modelled femoral fracture, compared with one lateral plate [9]. However, the implantation of the medial plate in addition to the lateral one using the traditional surgical technique makes the operation of bone osteosynthesis

[1 Kochish A.Iu., Belen'kii I.G., Sergeev G.D., Maiorov B.A. Anatomical and clinical justification of a minimally invasive technique for implantation an additional medial plate for bone osteosynthesis in patients with fractures of the distal femur. Genij Ortopedii, 2020, vol. 26, no 3, pp. 306-312. DOI 10.18019/1028-4427-2020-26-3-306-312 
very traumatic and may lead to postoperative complications, especially in high-energy injuries. Therefore, with bilateral bone fixation of the fractures under discussion, it is advisable to introduce the medial plate also by means of a minimally invasive technique. However, the technical feasibility of performing such an operation and, in particular, the safety of performing medial surgical mini-approaches has not been sufficiently studied, which limits the use of this technique in surgical practice.

Purpose of the study To substantiate the technique of minimally invasive implantation of an additional medial plate by means of applied topographic and anatomical studies and to test clinically the original method of minimally invasive osteosynthesis with two plates in patients with fractures of the distal femur.

\section{MATERIAL AND METHODS}

Our study was approved by the Local Ethics Committee at the Federal State Budgetary Institution at Vreden NRC for TO and included the topographic and anatomical part, performed on the basis of the Department of Operative Surgery with Topographic Anatomy of the Kirov Military Medical Academy and the clinical tests were carried out at trauma departments of St. Petersburg's Alexandrovskaya Hospital and Vsevolozhsk ICH.

An applied topographic and anatomical study was performed on 16 lower extremities of 9 unfixed corpses ( 5 male and 4 female) of people who died at the age from 54 to 72 years without any injuries or deformities in the hip area. The main purpose of this part of the study was to develop the surgical technique of minimally invasive osteosynthesis using a non-fixed anatomical material for implantation of a medial plate to the lower half of the femur from two mini-approaches and to assess its safety in relation to damage to large vessels and nerves of the thigh. It should also be noted that the technique of this original operation was the basis of our proposed method of minimally invasive osteosynthesis with two plates for multi-fragmented fractures of the distal femur, for which RF patent for invention No. 2665158 was obtained [10].

For topographic and anatomical experiments, a straight reconstructive plate, $197 \mathrm{~mm}$ long, was preliminarily modeled according to a plastic model of the right or left femur. Then, the necessary anatomical landmarks were made on the skin of the thigh of the anatomical object as follows. First, a straight line was drawn connecting the superior anterior iliac spine to the middle of the pubic articulation. Next, from the middle of this line, a straight line was drawn to the adductor tubercle of the medial condyle of the femur. The indicated line corresponded to the projection of the femoral artery onto the skin of the thigh.

Then, the lines of the supposed skin incisions for surgical approaches were marked on the skin on the medial and anteromedial surfaces of the thigh. A distal skin incision of $4 \mathrm{~cm}$ was marked from the level of the lower edge of the medial epicondyle of the femur upwards and parallel to the projection line of the femoral artery, $2 \mathrm{~cm}$ in front of it. A 5-cm long proximal medial skin incision line was also made $2 \mathrm{~cm}$ anterior to the projection line of the femoral artery and parallel to it at the level of the upper end of the plate, which was applied to the thigh (Fig. 1).

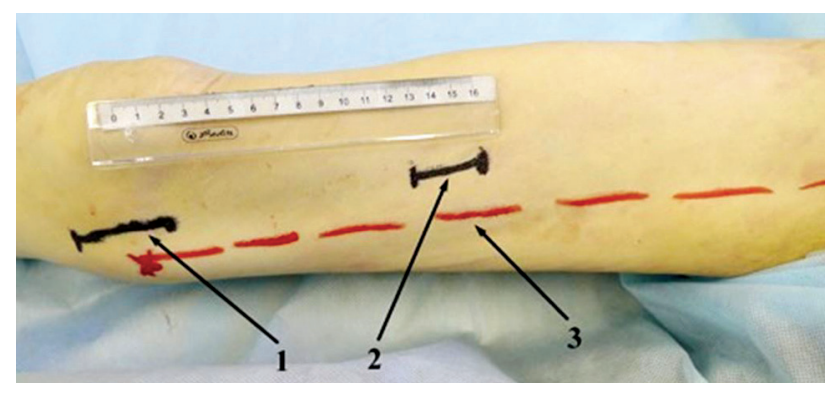

Fig. 1 Skin marks on the right thigh of the projection line of the femoral artery and skin incisions of two miniapproaches: 1 - line of distal surgical access; 2 - line of proximal surgical access; 3 - projection line of the femoral artery marked by a dotted line

The next step was a distal medial incision of the skin and soft tissues with a length of $4 \mathrm{~cm}$ along the marked line. Then, through this incision from the bottom up and epiperiosteally, a pre-modeled plate was introduced onto the anteromedial surface of the femur towards the superior anterior iliac spine until its distal end reached the lower edge of the distal incision made.

Next, at the level of the proximal end of the implanted plate on the anteromedial surface of the thigh, along the previously marked line, a proximal medial skin incision of $5 \mathrm{~cm}$ was made. The proper fascia of the thigh was exposed and the gap between the rectus and medial vastus muscles of the thigh was visualized. Then, by dissecting and separating the soft tissues in this space, the medial vastus and intermediate vastus of the femur were passed in between; the femur was exposed to visualize the proximal end of the inserted plate. Then, a metal retractor was inserted through the specified access under the posterior surface of the femur in order to prevent damage to the large blood vessels of the thigh with the screws fixing the plate by their insertion. Then, the medial plate was fixed with the screws with angular stability, $3.5 \mathrm{~mm}$ in diameter, two screws both proximally and distally (Fig. 2). 


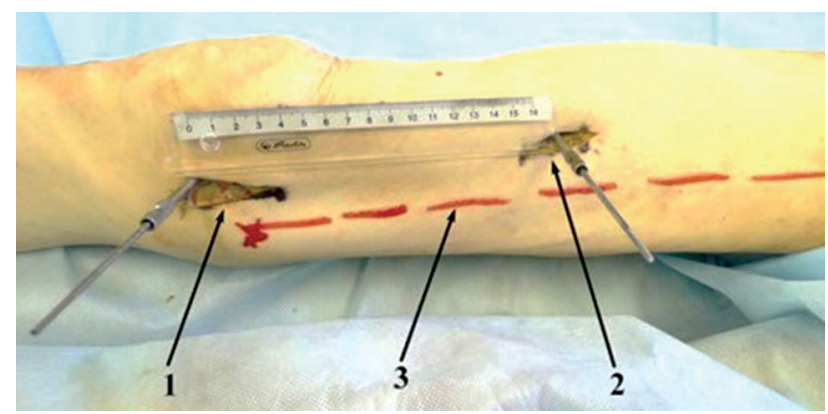

Fig. 2 View of the right thigh after minimally invasive implantation of the plate on the medial surface of the femur according to the method proposed: 1 - distal surgical approach; 2 - proximal surgical approach; 3 - projection of the femoral artery on the skin of the thigh outlined by a dotted line

The next step was precision preparation (Fig. 3), clarifying the relationship of the plate, implanted according to the minimally invasive method described above, with a number of important anatomical structures and making certain measurements using a caliper and a ruler. In particular, the minimum (shortest) distances from the plate to the femoral artery, the vein of the same name, and the saphenous nerve (n. Saphenus) were measured, as well as to the sites of origin of some branches of the femoral artery and to the tibial collateral ligament of the knee joint. In addition, the location of these formations in relation to the adductor tubercle on the medial epicondyle of the femur was determined by measurements. Subsequently, based on the data obtained, the average values of the measured distances and the corresponding standard deviations were calculated.

The clinical part of our work was carried out in the course of a prospective observational study and consisted in testing of the method of minimally invasive osteosynthesis with two plates implanted along the lateral and medial surfaces of the femur in patients with comminuted fractures of the distal femur of types 33-A3, 33-C2 and 33-C3 according to AO / ASIF classification with loss of medial stability. A total of 15 operations were performed in specialized trauma departments of two hospitals in nine men and six women aged 23 to 70 years (mean, $51.3 \pm 7.2$ years).

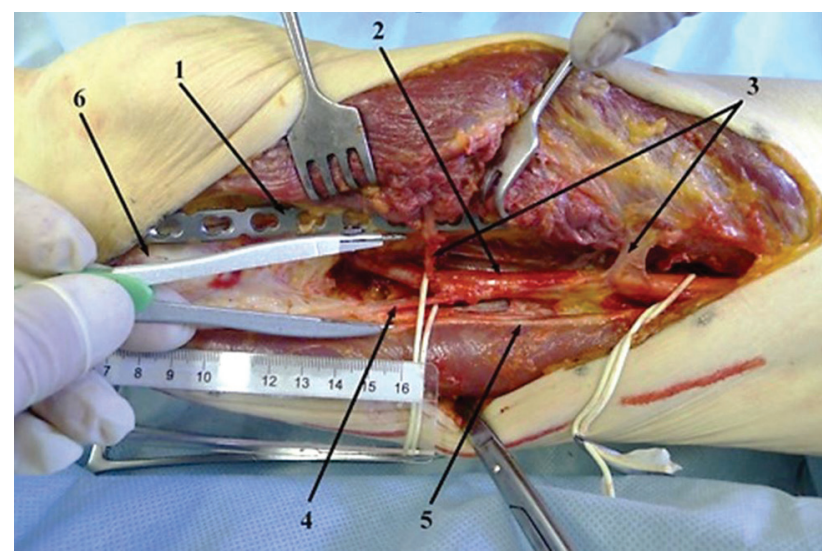

Fig. 3 Preparation of the right femur after minimally invasive installation of the medial plate in the lower half of the femur (the distance from the plate to the saphenous nerve (n. Saphenus) is measured with a caliper: 1 - installed plate; 2 - femoral artery; 3 - the muscular branches of the femoral artery, 4 - the descending knee artery; 5 - saphenous nerve; 6 - tibial collateral ligament of the knee joint

In the course of the steosynthesis operations, the technique of minimally invasive implantation of the medial plate and its fixation to the lower half of the femur, previously tested on anatomical material, was practically used, and the risks of damage to important anatomical structures (blood vessels and nerves) were studied and analyzed. In addition, the complications and outcomes of surgical treatment up to 12 months after the operations were studied. The functional assessment of treatment results was performed using the KSS scales (according to D.B. Kettelkamp et al., 1972) [11] and Lysholm (according to Y. Tegner, J. Lysholm, 1985) [12]. On the KSS scale, the results were considered excellent in the range of 83-103 points, good - 70-82, fair - 60-69, poor - less than 60 points. Similar ranges on the Lysholm scale were 91-100 points - excellent results, 84-90 - good, 65-83 - fair, less than 65 - poor.

The results of topographic and anatomical studies and the clinical data were compared with each other, as well as with the data in the publications of other authors. All quantitative data were subjected to statistical processing to determine the mean values and the corresponding standard deviations in Excel 2016 (Microsoft, USA).

\section{RESULTS}

With regard to the experiments on the anatomical material, it should be noted, first of all, that none of the 16 studied preparations of the lower extremities showed damage to important anatomical structures or direct contact of the plate with them. On the contrary, it was found that the plate installed to the lower half of the femur from two mini-approaches using the minimally invasive method proposed was always at a safe distance from the anatomical structures located on the medial surface of the thigh in its lower third. Thus, the minimum distances from the plate to the femoral artery and vein were 16 and $17 \mathrm{~mm}$, respectively, and the large branches of these vessels to the muscles of the anterior thigh group were always superficial and posterior to the plate implanted epiperiosteally and located directly along the periosteum of the femur. The plate was also far enough from the descending knee and medial superior knee arteries, at least 24 and $23 \mathrm{~mm}$, 
respectively. The minimum distances to the saphenous nerve (n. Saphenus) and to the tibial collateral ligament of the knee joint were also safe and measured 19 and $11 \mathrm{~mm}$, respectively. The results of the measurements are presented in Table 1. Based on the assessment of these results, it was concluded that it is possible to safely attach the medial plate to the lower half of the femur using the proposed method, provided that the technique of the developed operation is observed.

Among 15 patients who underwent surgery according to the method proposed, seven had a fracture of the distal femur due to low-energy trauma. Before the operation of osteosynthesis, they were treated with skeletal traction. In 8 patients, the fracture resulted from high-energy injuries. Upon admission, they underwent immobilization with an external fixation apparatus. Two of these patients had open fractures of types II and IIIA according to the Gustillo-Anderson classification. In four cases, the fractures were a component of polytrauma. These patients were treated according to the damage control protocol.

Osteosynthesis operations according to the method proposed were performed within four to 17 days from the moment of injury. The follow-up period varied from 6 to 12 months after the operation, but always exceeded the time of fracture healing. Clinical and radiological signs of fracture union were noted after 6 months in 11 of 15 patients (73.3\%), after 9 months in 12 of 14 patients (85.7\%), and after 1 year in all 11 patients $(100 \%)$. The range of motion in the knee joint, measured in degrees from full extension $\left(0^{\circ}\right)$, averaged $80 \pm 5.9^{\circ}\left(30-120^{\circ}\right)$ after 6 months, and $88 \pm 5.3^{\circ}\left(35-125^{\circ}\right)$ after 9 months and $96 \pm 4.8^{\circ}$ $\left(40-125^{\circ}\right)$ after 12 months. The functional results of treatment according to the used score rating scales KSS and Lysholm are presented in Tables 2 and 3.

Table 1

Average values of the minimum distances (MD) measured from the installed plate to the formations of the distal femur studied

\begin{tabular}{|c|c|c|c|c|}
\hline № & Distance & Minimum & Maximum & Mean values \\
\hline 1 & MD from plate to femoral vein (FV) & 16 & 32 & $24 \pm 5 \mathrm{~mm}$ \\
\hline 2 & MD from adduction tubercle (AT) to the point of measurement on FV & 112 & 139 & $124 \pm 8 \mathrm{~mm}$ \\
\hline 3 & MD from plate to femoral artery (FA) & 17 & 33 & $25 \pm 5 \mathrm{~mm}$ \\
\hline 4 & MD from AT to FA & 112 & 137 & $124 \pm 8 \mathrm{~mm}$ \\
\hline 5 & MD from plate to subcutaneous nerve (SN) & 19 & 37 & $27 \pm 5 \mathrm{~mm}$ \\
\hline 6 & MD from AT to SN & 106 & 137 & $123 \pm 8 \mathrm{~mm}$ \\
\hline 7 & $\begin{array}{l}\text { MP from the plate to the origin site of the descending knee artery (DKA) } \\
\text { from the femoral artery }\end{array}$ & 24 & 40 & $30 \pm 5 \mathrm{~mm}$ \\
\hline 8 & MD from adduction tubercle to the origin site of DKA & 115 & 144 & $132 \pm 9 \mathrm{~mm}$ \\
\hline 9 & $\begin{array}{l}\text { MD from the plate to the origin site of the medial superior knee artery } \\
\text { (MSKA) from femoral artery }\end{array}$ & 23 & 37 & $30 \pm 4 \mathrm{~mm}$ \\
\hline 10 & MD from AT to the site of MSKA origin & 64 & 100 & $79 \pm 10 \mathrm{~mm}$ \\
\hline 11 & $\begin{array}{l}\text { MD from the plate to the tibial collateral ligament of the knee joint } \\
\text { (TCLKJ) }\end{array}$ & 11 & 14 & $12 \pm 1 \mathrm{~mm}$ \\
\hline 12 & MD from AT to TCLKJ & 20 & 26 & $22 \pm 2 \mathrm{~mm}$ \\
\hline
\end{tabular}

Table 2

Qualitative assessment of functional results of treatment on KSS scale

\begin{tabular}{|l|c|c|c|}
\hline \multirow{2}{*}{ Treatment result } & \multicolumn{3}{|c|}{ Follow-up } \\
\cline { 2 - 4 } & 6 months & 9 months & 12 months \\
\hline Poor & $2(13 \%)$ & $1(7 \%)$ & $3(27 \%)$ \\
\hline Fair & $9(60 \%)$ & $4(29 \%)$ & $6(55 \%)$ \\
\hline Good & $4(27 \%)$ & $8(57 \%)$ & $2(18 \%)$ \\
\hline Excellent & $0(0 \%)$ & $1(7 \%)$ & $11(100 \%)$ \\
\hline Total & $15(100 \%)$ & $14(100 \%)$ & \\
\hline
\end{tabular}

Table 3

Qualitative assessment of functional results of treatment on Lysholm scale

\begin{tabular}{|l|c|c|c|}
\hline \multirow{2}{*}{ Treatment result } & \multicolumn{3}{|c|}{ Follow-up after osteosynthsis } \\
\cline { 2 - 4 } & 6 months & 9 months & 12 months \\
\hline Poor & $3(20 \%)$ & $1(7 \%)$ & $3(0 \%)$ \\
\hline Fair & $8(53 \%)$ & $5(36 \%)$ & $6(55 \%)$ \\
\hline Good & $4(27 \%)$ & $8(57 \%)$ & $2(18 \%)$ \\
\hline Excellent & $0(0 \%)$ & $0(0 \%)$ & $11(100 \%)$ \\
\hline Total & $15(100 \%)$ & $14(100 \%)$ & \\
\hline
\end{tabular}


In the course of 15 operations performed, there were no specific complications associated with the use of the method, as well as no damage to large vessels and nerves of the thigh. It confirms the safety of the technique and the correctness of the previous topographic and anatomical justifications. Postoperative complications were one case $(6.7 \%)$ of non-union 6 months after the operation which required delayed bone autologous plasty of the metadiaphyseal zone without reosteosynthesis, and one case $(6.7 \%)$ of early deep infection in a patient with a primary comminuted open (Gustillo-Anderson IIIA) 33-C3 fracture, which required the removal of the hardware and the transition to an external fixation device. In addition, two of our patients $(13.4 \%)$ were found to have instability of the lateral ligamentous apparatus of the knee joint, which required the use of a semi-rigid bracing. There were no cases of instability of fixation, migration or fractures of implants in our patients.

To demonstrate the successful clinical application of the method of the minimally invasive bone osteosynthesis proposed, we present the following clinical case. Patient B., 23 years old, a car driver, was injured in a road accident. He was brought to the hospital one hour after the injury and diagnosed with a multi-fragmented metaphyseal fracture of the distal part of the right femur with medial instability, 33-C3 according to the $\mathrm{AO}$ classification, fracture of the roof of the acetabulum with dislocation of the right femur, rupture of the pubic articulation. Taking into account the mechanism of injury, examination and treatment were performed in accordance with the polytrauma protocol. However, we focused in this clinical case only on the treatment of a fracture of the femur.

On admission, the fracture was temporarily fixed with an external fixation device (Fig. 4 a,b). On the fifteenth day after the injury, the operation of final fixation of the fracture of the right femur was performed. Osteosynthesis was performed using two lateral mini-approaches in the area of the lateral condyle and at the border of the upper and middle thirds of the diaphysis of the femur, $5 \mathrm{~cm}$ long, with the installation of the distal femoral plate in the mode of bridge fixation of the fracture. To increase the stability of fixation, a medial reconstructive plate was installed from two medial mini-approaches according to the method described above with fixation with two screws, $3.5 \mathrm{~mm}$ with angular stability, proximally and distally.
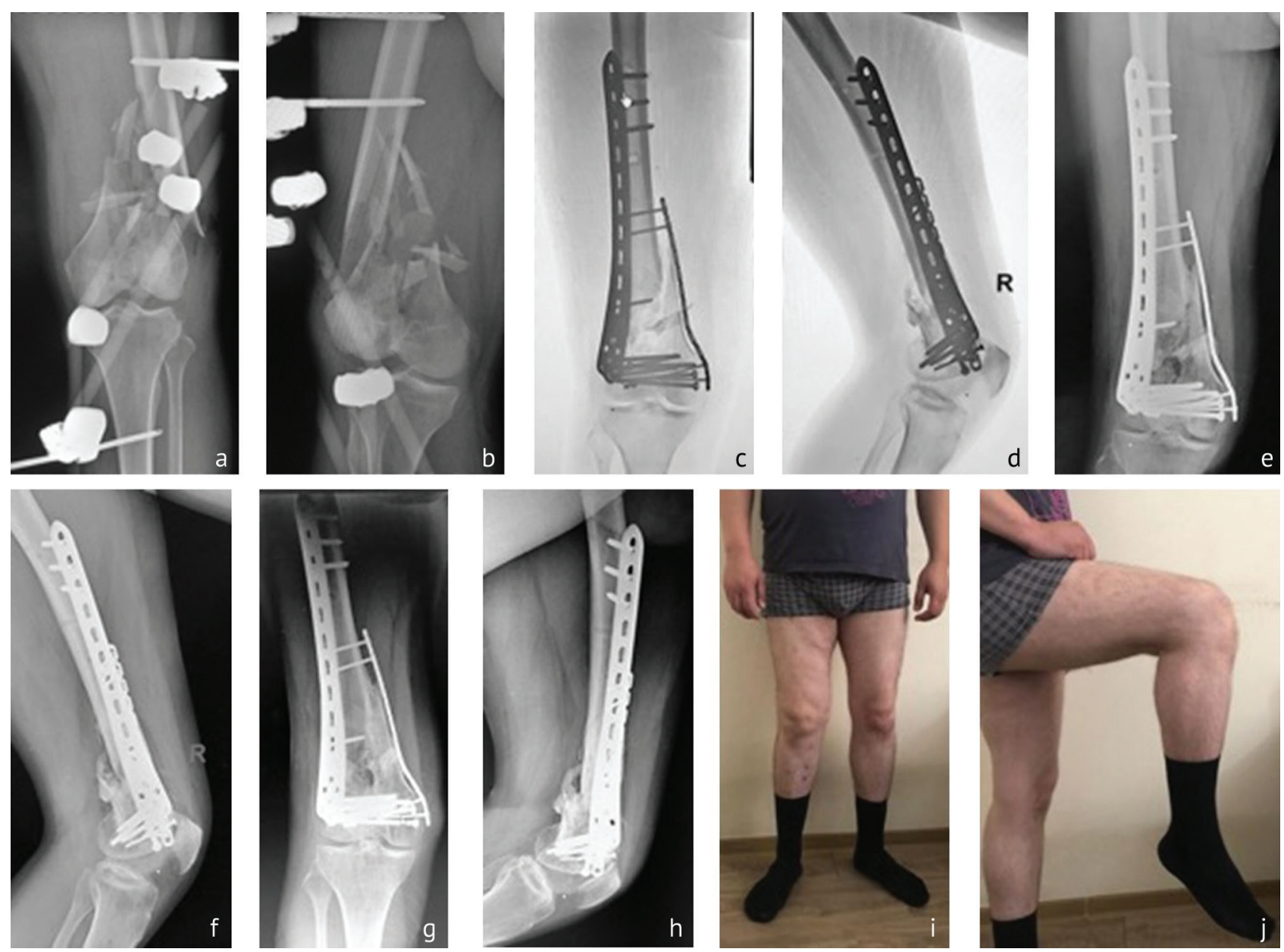

Fig. 4 Stages and result of osteosynthesis by the proposed method in patient B., 23 years old, with comminuted intra-articular fracture of the distal femur: $a, b-\mathrm{X}$-rays of the right femur and knee joint of patient B. in frontal and lateral projections after preliminary osteosynthesis with external fixation apparatus; $c, d$ - result of final surgical treatment, minimally invasive immersion osteosynthesis of the right femur with lateral and medial plates and screws with angular stability according to the proposed method; $e, f-\operatorname{control}$ $\mathrm{X}$-rays of the right femur 6 months after the operation: there are radiographic signs of fracture consolidation; $g, h-\mathrm{X}$-ray result of osteosynthesis a year after surgery with signs of callus remodeling; $i, j$ - functional result one year after osteosynthesis 
As a result of the operation, the axis and length of the segment were restored, rotational displacements were eliminated, and stable fixation of bone fragments was achieved (Fig. 4 c, d). Immobilization after surgery was not used. The wounds healed by primary intention. In the postoperative period, the patient did physiotherapy exercises, walked on crutches without support on the right lower limb. The axial load on this limb was restored three months after surgery.
After six months, clinical and radiological union of the fracture was observed (Fig. 4 e, f). The patient walked with full load on the right lower limb, there was no pain. One year after the operation, bone callus remodeling was noted (Fig. $4 \mathrm{~g}, \mathrm{~h}$ ). The function of the knee joint at that time-point: full active extension, active flexion up to $80^{\circ}$ in the absence of ligamentous apparatus instability (Fig. 4 i, j). The functional result one year after the operation was assessed using the Lyscholm scales (84 points) and KSS (85 points) and was rated good.

\section{DISCUSSION}

The problem of osteosynthesis quality in fractures with medial instability is not exclusive for the distal femur and is typical for all eccentrically loaded limb segments. Theoretically, one of the simplest solutions to this problem could be the transition from internal extramedullary to intramedullary osteosynthesis, which significantly reduces the distance from the fixator axis to the anatomical axis of the limb and, accordingly, reduces the load on the implant. In particular, this is how the problem of medial stability is solved in intertrochanteric fractures of the femur with a fracture of the lesser trochanter. In such cases, the gamma-shaped intramedullary fixator provides greater stability compared to extramedullary fixators [13].

A similar situation occurs in fractures of the proximal humerus [14]. However, ensuring medial stability in fractures of the upper limb bones is less important due to the lack of axial load on them by standing and walking. The distal femur has a relatively small amount of soft tissue surrounding the knee joint. However, the functional significance of the knee joint is quite high, and its functioning disorders significantly affect the quality of life. That is why, a thorough reconstruction of the anatomy of the articular surface and restoration of the femoral axis are necessary conditions in fractures of the distal femur.

Moreover, the biological aspects of osteosynthesis should not be ignored since most comminuted fractures in the region under discussion are of a high-energy nature. Thus, the simplest solution to the problem of medial instability, osteosynthesis with two plates, using traditional surgical approaches in the distal femur area can lead to excessive surgical trauma and complications associated with soft tissues and fracture union.

Intramedullary osteosynthesis in multiple fractures of the distal femur often cannot be implemented technically or requires an extended surgical approach to reduce the articular component of the fracture, which significantly decreases the biological value of this method. Theoretically, in such fractures, osteosynthesis with one medial plate would be possible but then it would have to be of sufficient length and reach the upper third of the femur. In this case, its proximal fixation would be technically difficult and could lead to damage to the major vessels of the thigh.
With this fact in mind, it seems logical that an approach with which medial stability in the lower third of the diaphysis of the injured femur is achieved by osteosynthesis with two plates; and the invasiveness of the operation is reduced due to the minimally invasive technique of their implantation. There is no need to use a long medial plate. It is possible to fix its proximal end at the level of the middle third of the femur, and to perform the corresponding surgical mini-approach through the intermuscular spaces between the abdomens of the rectus and medial vastus muscles, and deeper, between the intermediate vastus and medial vastus muscles of the thigh, which lack large blood vessels. It should also be noted that previous experimental studies showed that such osteosynthesis significantly increases the resistance of the "implant-bone" system to cyclic axial load [9].

The present work demonstrates the technical feasibility of the operation and its safety in regard to the damage to clinically significant vessels and nerves of the thigh. The data obtained allow us to conclude that during epiperiosteal implantation of a pre-modeled plate on the medial surface of the femur using the proposed method significant anatomical structures of the distal femur cannot be injured being at a sufficient distance from the plate and from the site of surgical approaches along the medial surface of the thigh. For example, the minimum distance from the plate to the femoral artery and vein varies from 16 to $33 \mathrm{~mm}$, and these vessels themselves are located outside the zones of minimally invasive surgical approaches, and their damage in the course of the intervention is practically excluded.

In the course of the study, it was also found that the branches of the femoral artery running to the lateral vastus muscle of the thigh extend posteriorly from the plate, fixed on the bone, and are located more superficially. It means that they cannot be injured its epiperiosteal insertion and by performance of the two surgical approaches, described above. Topographic and anatomical studies also confirmed that the spaces between the rectus and medial vastus muscles, as well as between the medial and intermediate vastus muscles, through which the proximal medial approach to the femur is carried out, do not contain 
large vessels and are safe in terms of the risk of their injury.

It should also be emphasized that the practical feasibility and safety of the proposed method of minimally invasive osteosynthesis with two plates for fractures of the distal femur with loss of medial stability were fully confirmed during its clinical tests in 15 patients. It allows, in our opinion, its wider clinical approbation.

\section{CONCLUSION}

Thus, our anatomical and clinical study allows, in our opinion, concluding that the proposed method of minimally invasive osteosynthesis with two plates for fractures of the distal femur is feasible. Its technically correct implementation is safe since it does not lead to damage to significant anatomical structures of the thigh. The proposed method is promising for treating patients with medially unstable DF fractures and its wider clinical testing is possible. However, the exact indications for the use of a new osteosynthesis method should be finally determined after the accumulation of a sufficient clinical material, alongside with the study of its effectiveness and safety in comparison with other possible options for osteosynthesis.

\section{REFERENCES}

1. Ehlinger M., Ducrot G., Adam P., Bonnomet F. Distal femur fractures. Surgical techniques and a review of the literature. Orthop. Traumatol. Surg. Res., 2013, vol. 99, no. 3, pp. 353-360. DOI: 10.1016/j.otsr.2012.10.014.

2. Martinet O., Cordey J., Harder Y., Maier A., Bühler M., Barraud G.E. The epidemiology of fractures of the distal femur. Injury, 2000, vol. 31, no. Suppl. 3, pp. C62-C63. DOI: 10.1016/s0020-1383(00)80034-0.

3. Kolmert L., Wulff K. Epidemiology and treatment of distal femoral fractures in adults. Acta Orthop. Scand., 1982, vol. 53, no. 6, pp. 957-962. DOI: 10.3109/17453678208992855.

4. Batchelor E., Heal C., Haladyn J.K., Drobetz H. Treatment of distal femur fractures in a regional Australian hospital. World J. Orthop., 2014, vol. 5, no. 3, pp. 379-385. DOI: 10.5312/wjo.v5.i3.379.

5. Zlowodzki M., Bhandari M., Marek D.J., Cole P.A., Kregor P.J. Operative treatment of acute distal femur fractures: systematic review of 2 comparative studies and 45 case series (1989 to 2005). J. Orthop. Trauma, 2006, vol. 20, no. 5, pp. 366-371. DOI: 10.1097/00005131200605000-00013.

6. Crist B.D., Della Rocca G.J., Murtha Y.M. Treatment of acute distal femur fractures. Orthopedics, 2008, vol. 31, no. 7, pp. 681-690. DOI: 10.3928/01477447-20110505-08.

7. Rüedi T.P., Buckley R., Moran C.G. AO Principles of Fracture Management. Stuttgart, Thieme, 2007,1106 p.

8. Briffa N., Karthickeyan R., Jacob J., Khaleel A. Comminuted supracondylar femoral fractures: a biomechanical analysis comparing the stability of medial versus lateral plating in axial loading. Strategies Trauma Limb Reconstr., 2016, vol. 11, no. 3, pp. 187-191. DOI: $10.1007 / \mathrm{s} 11751-016-0268-0$.

9. Belenkii I.G., Sergeev G.D., Maiorov B.A., Semenov S.G., Benin A.V. Eksperimentalnoe i teoreticheskoe obosnovanie dvukhkolonnoi teorii osteosinteza pri perelomakh distalnogo otdela bedrennoi kosti [Experimental and theoretical justification of the two-column theory of osteosynthesis for distal femoral fractures]. Travmatologiia i Ortopediia Rossii, 2017, vol. 23, no. 3, pp. 86-94. (in Russian)

10.Belenkii I.G, Semenov S.G., Kochish A.Iu., Sergeev G.D., Maiorov B.A., Benin A.V. Sposob maloinvazivnogo osteosinteza pri mnogooskolchatykh perelomakh distalnogo otdela bedrennoi kosti [The way of little-invasive osteosynthesis for multi-fragmental fractures of the distal femur]. Patent RF, no. 2665158, 2017. (in Russian)

11.Kettelkamp D.B., Chao E.Y. A method for quantitative analysis of medial and lateral compression forces at the knee during standing. Clin. Orthop. Relat. Res., 1972, vol. 83, pp. 202-213. DOI: 10.1097/00003086-197203000-00037.

12.Tegner Y., Lysholm J. Rating systems in the evaluation of knee ligament injuries. Clin. Orthop. Relat. Res., 1985 , no. 198 , pp. 43-49.

13.Marmor M., Liddle K., Pekmezci M., Buckley J., Matityahu A. The effect of fracture pattern stability on implant loading in OTA type 31-A2 proximal femur fractures. J. Orthop. Trauma, 2013, vol. 27, no. 12, pp. 683-689. DOI: 10.1097/BOT.0b013e31828bacb4.

14.Jung W.B., Moon E.S., Kim S.K., Kovacevic D., Kim M.S. Does medial support decrease major complications of unstable proximal humerus fractures treated with locking plate? BMC Musculoskelet. Disord., 2013, vol. 14, pp. 102. DOI: 10.1186/1471-2474-14-102.

Received: 13.01.2020

\section{Information about the authors:}

1. Alexander Iu. Kochish, M.D., Ph.D., Professor, Vreden National Medical Research Center of Traumatology and Orthopedics, Saint Petersburg, Russian Federation, Medical Military Academy named after S.M. Kirov, St. Petersburg, Russian Federation, Email: auk1959@mail.ru

2. Igor G. Belen'kii, M.D., Ph.D.,

Pavlov First St. Petersburg State Medical University, Saint Petersburg, Russian Federation,

Email: belenkiy.trauma@mail.ru

3. Gennady D. Sergeev,

Vreden National Medical Research Center of Traumatology and Orthopedics, Saint Petersburg, Russian Federation

4. Boris A. Maiorov, M.D., Ph.D.,

Pavlov First St. Petersburg State Medical University, Saint Petersburg, Russian Federation,

Vsevolozhsk clinical inter-district hospital, Vsevolozhsk, Leningrad region, Russian Federation,

Email: MayorovBA@vkmb.ru,bmayorov@mail.ru 